Conclusion: Patients with systemic sclerosis positive for anti-U1RNP differ in the predominance of inflammatory musculoskeletal manifestations and frequent combination with Sjogren's syndrome and overlaps. Highly positivity for anti-U1RNP is accompanied by a persistent increase in RF, anti-Ro, anti-dsDNA

Disclosure of Interests: None declared

DOI: 10.1136/annrheumdis-2020-eular.5678

\section{AB0610 SEASONAL VARIATION IN IDIOPATHIC INFLAMMATORY MYOPATHIES INCIDENCE AND PRESENTATION: A RETROSPECTIVE STUDY IN BEIJING AND HONG KONG}

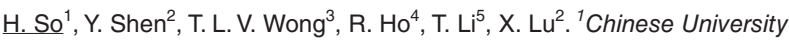
of Hong Kong, New Territories, Hong Kong (SAR); ${ }^{2}$ China Japan Friendship Hospital, Beijing, China; ${ }^{3}$ Kwong Wah Hospital, Kowloon, Hong Kong (SAR); ${ }^{4}$ Queen Elizabeth Hospital, Kowloon, Hong Kong (SAR); ${ }^{5}$ Queen Mary Hospital, Hong Kong, Hong Kong (SAR)

Background: Seasonal patterns of disease onset and severity in idiopathic inflammatory myopathies (IIMs) as a whole are conflicting [1-3]. In recent years, over 10 myositis-specific antibodies (MSAs) have been identified. They are able to divide patients into homogenous subgroups and inform on prognosis [4].

Objectives: The objective of the study was to investigate the seasonal variation of onset of IIMs characterised serologically.

Methods: This was a multi-centred retrospective observational study. Consecutive Chinese patients with IIMs admitted to the rheumatology wards of the participating major regional hospitals in Beijing and Hong Kong from July 2013 to June 2018 were recruited. The diagnosis of IIMs was based on the Bohan and Peter's criteria with definite or probable cases being included [5]. Patients with clinically amyopathic disease must have the typical Gottron's papules or heliotrope rash as determined by rheumatologists or dermatologists, and with no symptoms or signs of muscle involvement according to Sontheimer [6]. Patients with juvenile myositis, inclusion body myositis, cancer-associated myositis and myositis associated with other connective tissue disease were excluded. A commercial line blot immunoassay kit (EUROLINE) was used to detect the MSAs.

Results: All together 495 patients were studied. The mean age of the patients at disease onset was 48.1 years (S.D. 13.3). There was a female predominance (68.3\%). The subgroups of IIMs were: dermatomyositis (61.0\%), polymyositis $(21.8 \%)$, clinically amyopathic dermatomyositis $(12.9 \%)$, immune mediated necrotising myopathy $(3.8 \%)$ and nonspecific myositis $(0.4 \%)$. No particular seasonal pattern in disease onset was observed in IIM patients as a whole (Figure 1) or in any classical subgroups. However, significantly more patients with any one MSA had their disease started in the first half of the year $(p=0.007)$ as shown in Figure 2. Patients with either anti-synthetase or anti-MDA5 antibodies, which are associated with interstitial lung disease, had more frequent disease onset from November to February, which might coincide with the local flu season. It was also found that MSA positivity was associated with infection of the patient $(p=0.005)$. Further analyses showed that patients with MSAs which are typically associated with severe skin disease (MDA5, TIF1g, NXP2, SAE) had more hospitalisation from April to September where excessive sun exposure is expected. There were no major differences between the Beijing and Hong Kong subgroups.

Conclusion: Apparent seasonal patterns were noticed in our ethno-serologically defined IIM patients. Certain environmental factors, particularly infection or UV exposure, could be potential triggers. Our findings could shed light on the identification of etiologic factors and enhance our understanding of disease pathogenesis.

References:

[1] Manta P, Kalfakis N, Vassilopoulos D. Evidence for Seasonal Variation in Polymyositis. Neuroepidemiology 1989;8:262-265.

[2] Phillips BA, Zilko PJ, Garlepp MJ, et al. Seasonal occurrence of relapses in inflammatory myopathies: a preliminary study. J Neurol 2002;249:441-4.

[3] Lefe R, Burgess S, Miller F, et al. Distinct Seasonal Pattern in The Onset of Adult Idiopathic Inflammatory Myopathy in Patients with Auto Antibodies Anti-Jo-1 and Anti-Signal Recognition particle. Arthritis and Rheumatism 1991;34(11):1391-1396.

[4] Tansley SL, Betterridge ZE, McHugh NJ. The diagnostic utility of autoantibodies in adult and juvenile myostis. Curt Opin Rheumatol 2013;25(6):772-777.

[5] Bohan A, Peter JB. Polymyositis and dermatomyositis. N Engl J Med 1975;292:344-347.

[6] Sontheimer RD. Clinically myopathic dermatomyositis: what can we now tell our patients? Arch Dermatol 2010;146(1):76-80.
Figure 1.Disease onset of myositis patients.

50

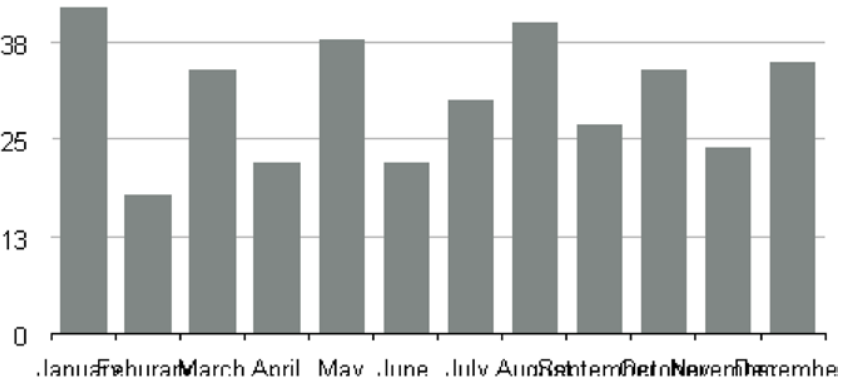

Figure 2. Disease onset of myositis patients with or without myositis specfic antibodies (MSA)

40

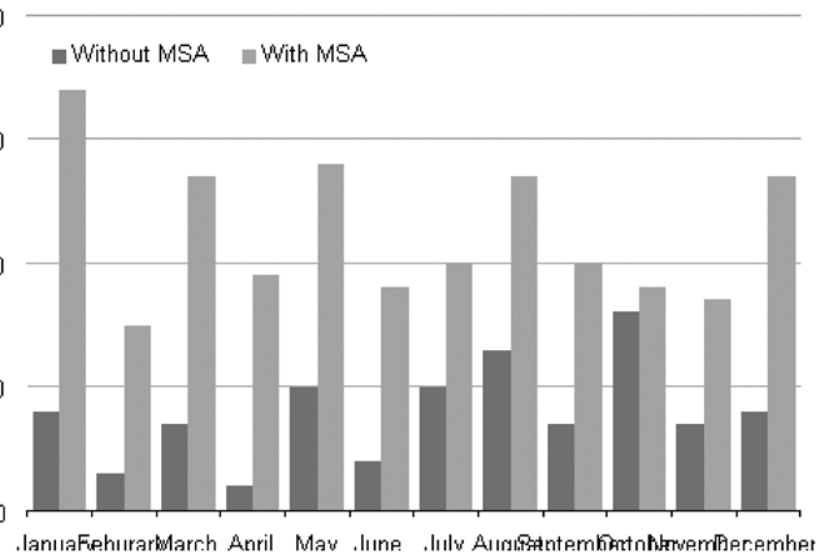

Disclosure of Interests: None declared

DOI: 10.1136/annrheumdis-2020-eular.5882

\begin{tabular}{|l|}
\hline AB0611 \\
STRAIN ANALYSIS OF THE RIGHT VENTRICLE USING \\
2D-SPECKLE TRACKING ECHOCARDIOGRAPHY IN A \\
COHORT OF PATIENTS WITH SYSTEMIC SCLEROSIS
\end{tabular}

A. Spinella ${ }^{1}$, P. Macripo, ${ }^{1}$, E. Cocchiara ${ }^{1}$, E. Galli ${ }^{1}$, F. Lumetti ${ }^{1}$, L. Magnani ${ }^{2}$, F. Coppi ${ }^{1}$, A. V. Mattioli ${ }^{1}$, R. Rossi ${ }^{1}$, G. Boriani ${ }^{1}$, C. Salvarani ${ }^{1}$, D. Giuggioli ${ }^{1}$.

${ }^{1}$ Policlinico of Modena University Hospital of Modena, Modena, Italy;

${ }^{2}$ Arcispedale Santa Maria Nuova, Reggio Emilia, Italy

Background: Systemic Sclerosis (SSc) is a rare and life-threatening connective tissue disease with multiple organ impairment. Cardio-pulmonary involvement is common: pulmonary fibrosis, pulmonary hypertension $(\mathrm{PH})$, and electrical disorders are the most serious complications and causes of increased mortality. Objectives: We evaluated features related with the onset and development of $\mathrm{PH}$ in a cohort of SSc patients. We further studied ecocardiographic abnormalities, by means of 2D-speckle tracking echocardiography (STE) with specific reference to the right ventricular strain measure (RV-strain).

Methods: We analyzed data from 50 SSc patients (pts) referred to our University-based Rheumatology Centre and SSc Unit from January 2007 to June 2019 (F/M 45/5; Ic/dcSSc 45/5; mean age 59.20 14.357 years; mean disease duration $12.08 \pm 8.75$ years). All pts underwent general and cardio-pulmonary assessment in our Cardio-Rheumatology Clinic. The following parameters were considered: blood exams, in particular inflammation indexes, uric acid test and serum autoantibodies; pulmonary function tests; high resolution scan of the lungs (HRCT); standard electrocardiogram (ECG) and RV-strain measured by 2D-STE. These examinations were performed according to clinical picture and current methodologies. We compared SSc subjects with (10/50) and without (40/50) PH diagnosis during follow-up regardless of treatments.

Results: SSc pts with $\mathrm{PH}$ didn't show significant alterations concerning RV-strain if compared with pts without PH ( $\mathrm{p}=0.707)$. Nevertheless, RV-strain value was modified in relation to TAPSE alterations in all pts but this data correlated with right ventricular dilatation only in $\mathrm{PH}$ subjects. Furthermore, interesting significant values about dilatation of right and left atria $(p=0.007, p=0.048)$, dilatation of inferior vena cava $(p=0.037)$ and right ventricle $(p=0.023)$ were observed. Left ventricular hypertrophy $(\mathrm{p}=0.012)$ as well as valvular insufficiencies (mitral and aortic) were more frequent in $\mathrm{PH}$ group too $(p=0.016)$. These pts showed higher incidence of 